UDC 316.6

LBC 60.54

\title{
FAMILY AS A SOURCE OF INTERGENERATIONAL SOLIDARITY. SPECIAL ATTITUDE TO SENIOR SOCIETIES: EXAMPLE OF SPAIN
}

\author{
Belén Zárate Rivero \\ International University of Catalonia (UIC Barcelona), Barcelona, Spain \\ Montserrat Gas-Aixendri \\ International University of Catalonia (UIC Barcelona), Barcelona, Spain
}

\begin{abstract}
Methodological basis of the study includes approaches to intergenerational solidarity. The following issues were studied: stability of family values, processes of mutual assistance and solidarity between different generations, family impact on the individual well-being of its members, main conflicts arising in the family, family and friendly relations, attitude to other generations, gratitude and sense of equality, health, leisure, using ICT, availability of paid work, participation in social and political as well as volunteer activities, social capital, idea of oldness etc. The quota sampling (sample size - 629 interviews) was based on the distribution of elderly population aged 65 to 74 years in Spain (including the islands). The only distortion of the theoretical sampling was inability to build it on quotas that would accurately reflect the distribution of the selected age population with children, since official statistic data are unavailable to the public on the number of parents with children by age group. Locations of interviews were chosen randomly, taking into account the distribution of the elderly population aged 65 to 74 years across the country, divided into autonomous regions (17) and locations (5 types). Selection process was based on 85 indicators. Territorial areas and within them streets for interviews were also randomly selected according to each indicator within the selected locations. Numbers of buildings and floors were chosen randomlyto search for individuals matching the sampling (meeting the quota requirements), and then they were interviewed. The following conclusion was made: the value of individualism is growing in society, family solidarity helps older people to overcome many psychological and social problems.

Key words: institute of family, social groups, family solidarity, intergenerational solidarity, solidarity index, statistics, elderly people.
\end{abstract}

УДК 316.6

ББК 60.54

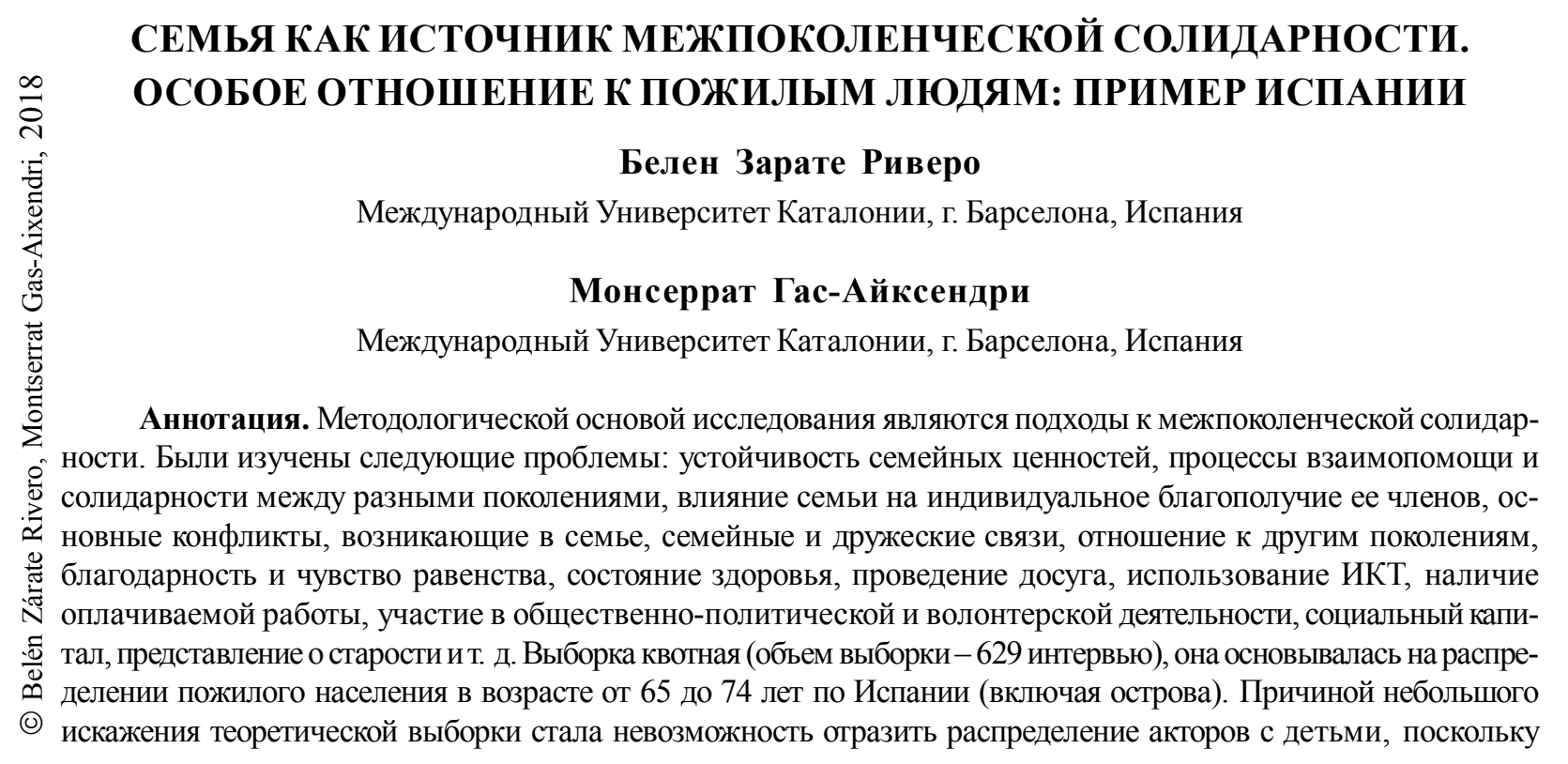


официальная статистика не предоставляет в открытом доступе данные по возрастным группам, учитывающие количество родителей с детьми, что не позволяет использовать эту информацию при построении квотной выборки. Случайным образом, с учетом распределения пожилого населения в возрасте от 65 до 74 лет по территории страны с разделением на автономные регионы (17) и места проведения (5 типов), были выбраны места проведения опроса. В целом процесс отбора базировался на 85 показателях. Среди выбранных мест проведения опроса случайным образом по каждому показателю были выбраны территориальные участки и, среди последних, улицы для проведения опросов. Случайным образом выбирались номера домов и этажей для поиска лиц, соответствующих выборке (удовлетворяющих требованиям квоты), и проводился их опрос. Сделан следующий вывод: несмотря на то что в обществе возрастает значимость индивидуализма, семейная солидарность помогает пожилым людям преодолеть многие проблемы психологического и социального характера.

Ключевые слова: институт семьи, социальные группы, семейная солидарность, межпоколенческая солидарность, индекс солидарности, статистика, пожилые люди.

CDU 316.6

BCB 60.54

LA FAMILIA COMO FUENTE DE LAS RELACIONES

INTERGENERACIONALES DE SOLIDARIDAD. APLICACIONES ESPECIALES PARA EMPRESAS MAYORES: EL EJEMPLO DE ESPAÑA

\author{
Belén Zárate Rivero \\ Universitat Internacional de Catalunya (Barcelona, España), Barcelona, España \\ Montserrat Gas-Aixendri \\ Universitat Internacional de Catalunya (Barcelona, España), Barcelona, España
}

\begin{abstract}
Anotación. Las bases metodológicas del estudio son los enfoques de la solidaridad intergeneracional. Se estudiaron los siguientes problemas: la estabilidad de los valores familiares, los procesos de asistencia mutua y solidaridad entre diferentes generaciones, la influencia de una familia en el bienestar individual de sus miembros, los principales conflictos en la familia, la familia y las amistades, las actitudes hacia otras generaciones, la gratitud y el sentido de igualdad, salud actividades de ocio, uso de las TIC, trabajo remunerado, participación en actividades sociales, políticas y voluntarias, capital social, ideas sobre la vejez etc. La muestra de cuota (tamaño de la muestra - 629 entrevistas) se basó en la distribución de la población anciana de 65 a 74 años en España (incluidas las islas). La única distorsión de la muestra teórica fue la incapacidad de construirla de acuerdo con cuotas que reflejarían con precisión la distribución de la población de la edad seleccionada con niños vivos, ya que las estadísticas oficiales no tienen datos de acceso abierto sobre el número de padres con hijos vivos por grupo de edad. Las ubicaciones de la encuesta se seleccionaron al azar, teniendo en cuenta la distribución de la población anciana de 65 a 74 años en todo el país, dividida en regiones autónomas (17) y ubicaciones (5 tipos). En general, el proceso de selección se basó en 85 indicadores. Entre los lugares seleccionados para cada indicador, también al azar, se seleccionaron los sitios territoriales y, entre estos últimos, las calles para realizar encuestas. Los números de casas y pisos se seleccionaron al azar para buscar individuos que coincidían con la muestra (cumpliendo con los requisitos de cuota), y se encuestaron. Se hizo la siguiente conclusión: el valor del individualismo es cada vez mayor en la sociedad, la solidaridad familiar ayuda a las personas mayores a superar muchos de los problemas psicológicos y sociales.
\end{abstract}

Palabras clave: institución familiar, grupos sociales, solidaridad familiar, solidaridad intergeneracional, índice de solidaridad, estadísticas, personas mayores.

\section{Introducción: el trabajo del instituto de estudios superiores de la familia}

Es un placer para mí presentar una muestra de la investigación del Instituto de Estudios Superiores de la Familia, que tengo el honor de dirigir en la Universidad Internacional de Cataluña (Barcelona, España).

Para ponerles en antecedentes y enmarcar mejor mi intervención, permítanme hablarles brevemente sobre el trabajo que desarrolla nuestro Instituto y cuenta con diversas líneas de investigación: la primera línea de investigación, 
con la que iniciamos nuestro trabajo hace ya más de 15 años, se ocupa de la educación familiar en la que estudiamos desde el punto de vista de la antropología filosófica, de la educación familiar (pedagogía familiar), de la sociología y de la psicología, las relaciones familiares como relaciones que educan y apoyan el desarrollo integral de las personas.

El Instituto imparte diversos cursos de Postgrado en diversas áreas:

- postgrado en Filosofía y Pedagogía Familiar (se denomina Matrimonio y Educación Familiar), que se imparte on-line en lengua española desde 2005; en lengua inglesa desde 2008; en 2018 se comenzará a impartir también en lengua rusa.

- postgrado en Consultoría y Orientación Familiar, dirigido a formar profesionales que puedan aconsejar y apoyar a las familias que tienen alguna dificultad no patológica. También podrán derivar a los profesionales de la psicología y de la medicina en caso de patologías.

El trabajo del Instituto en esta área cuenta también con una dimensión práctica, que trata de aplicar los conocimientos a la orientación familiar, a la resolución de conflictos en el ámbito de la familia, y a la terapia familiar.

Junto a la Facultad de Psicología, recientemente creada en nuestra Universidad, colaboramos en la marcha de un Centro Universitario de Apoyo Psicológico a las personas y a las familias. Se trabaja a tres niveles: 1 - la promoción de la salud; 2 - el diagnóstico y la resolución de dificultades en las relaciones familiares que no provienen de una patología; 3 terapia en caso de patologías.

La segunda línea de investigación se refiere a la protección de la infancia y su conexión con las políticas de familia, a través de la Childcare and Family Policies Chair financiada por la Fundación Joaquim Molins Figueras. En esta línea se están trabajando.En este ámbito se ha creado un programa de formación: postgrado en Políticas Sociales de Familia, para la formación de Expertos en este campo que trabajen desde las administraciones públicas, las empresas o las entidades que trabajan con familias.

La tercera área de investigación tiene que ver con el estudio de la solidaridad familiar a través de la Cátedra IsFamily Banco Santander. Sobre esta línea desarrollaré mi intervención, hablándoles de uno de nuestros principales trabajos. Pero antes permítanme completar la explicación sobre el Instituto hablando de nuestra base conceptual y metodológica.

Marco conceptual: el trabajo del Instituto se mueve en el paradigma de la teoría relacional elaborada por el sociólogo italiano P. Donati [4, 5]. Este marco teórico ha sido probado en numerosas investigaciones empíricas en el campo de la vida familiar, la sociología del capital social, de la ciudadanía, del estado de bienestar y de las políticas sociales, entre otros. El marco metodológico del estudio se basa tambien en las obras de S. Arber [1], V. L. Bengtson y R.E.L. Roberts [2], R.H. Binstock [3], I. Jönsson [6], M.T. López López [7], K. Lüscher [8], G. Meil [9], E. Scabini y G. Rossi [10].

La teoría relacional de la sociedad consiste en observar que cualquier fenómeno o formación social es relación y está hecha de relaciones, que distinguen la forma y los contenidos de cada sociedad específica. La relación social debe entenderse, no como una realidad accidental, secundaria o derivada de otros individuos o sistemas, sino como realidad sui generis. El Profesor Pierpaolo Donati considera que la familia debe ser estudiada desde un punto de vista interno y a través del paradigma relacional, pues sólo de esta forma se puede conocer el sentido que ésta tiene para los demás subsistemas sociales. Donati no sólo ha reflexionado sobre la familia como relación, sino también sobre la familia como sujeto de relaciones con respecto al Estado y a los demás agentes de la sociedad. Por eso su contribución científica en el campo de las políticas familiares es muy relevante. Donati aporta un nuevo planteamiento para el análisis de la sociedad, a través de la propia familia; y otorga a esta institución la llave para entender y afrontar las paradojas de la sociedad contemporánea.

\section{La familia como ámbito de relaciones de solidaridad}

La Cátedra Is Family Santander tiene como objetivo estudiar la familia como ámbito de solidaridad intergeneracional, capaz de adaptarse al cambio y de dar apoyo a sus miembros. Los estudios que promueve la cátedra consideran la familia como lugar de transferencia de recursos entre generaciones, y se centran en algunos de sus aspectos fundamentales: la economía, la salud, 
el cuidado de las personas y la educación. Nuestra investigación está dirigida a encontrar soluciones a los problemas planteados por la Organización Mundial de la Salud [11]. La investigación está también dirigida a proponer políticas sociales que faciliten que las familias logren realizar estos procesos intergeneracionales.

La Cátedra pretende crear un conjunto de conocimientos y de acciones encaminados a aprovechar de modo beneficioso el potencial de la intergeneracionalidad en la familia, como motor de cambio social.

La solidaridad intergeneracional en la familia la compleja red de relaciones que configuran la vida de las personas en su contexto familiar. El concepto de "solidaridad familiar intergeneracional" se basa en seis elementos que miden la interacción padres-hijos desde diferentes puntos de vista: afecto, asociación, consenso, intercambio de recursos, solidaridad normativa (percepción de obligación que vincula al cuidado) y oportunidades de interacción [2, pp. 856-870].

\section{El estudio padres mayores, generaciones y solidaridad familiar}

El estudio "Padres mayores, generaciones y solidaridad familiar" se ha realizado con una metodología sociológica, pero está abierto a profundizaciones y desarrollos desde otras ciencias, principalmente la Psicología.

\subsection{Aspectos metodológicos:}

a) Instrumento: El cuestionario busca medir a través de 99 preguntas las diversas dimensiones de la solidaridad intergeneracional en el ámbito familiar, explorando también otros temas: red familiar y de amistad, actitudes hacia otras generaciones, agradecimiento y sentimientos de equidad, condiciones de salud y hábitos de ocio, uso de las TIC, tener un trabajo retribuido, la participación en actividades de voluntariado y en la esfera sociopolítica, capital social, valores, percepción de la condición anciana, ingresos y situación económica.

b) Muestra: El trabajo de campo, (noviembre-diciembre de 2016), ha permitido recoger 629 entrevistas en una muestra por cuotas de residentes de edad comprendida entre 65 y 74 años con hijos vivos en toda España. En total, en el 2015 había en el país 2283406 residentes de edad incluida entre 65 y 74 años. La muestra usada finalmente para los análisis se compone de 608 casos válidos.

c) Objetivos:

- el primer objetivo ha sido describir e interpretar las dimensiones de la solidaridad intergeneracional en España: cantidad y calidad de las relaciones, recursos intercambiados, objetivos individuales, normas y valores dentro de la familia - y que se extienden a otras esferas sociales relevantes (i.d. redes de vecinos y de amigos). Unas variables específicas han sido incluidas en el cuestionario para este fin.

- un segundo objetivo era analizar estadísticamente esos datos a la luz de la cohesión familiar intergeneracional: el afecto, los acuerdos generales, los recursos compartidos, la fuerza de las normas familiares, y las oportunidades que se dan en esa misma interacción analizadas por Bengtson and Roberts como elementos que, interrelacionados, explican la solidaridad familiar intergeneracional [2, pp. 856-870].

d) Características:

- se trata de la única encuesta realizada en España basada en una muestra nacional de la población -muestreada ad hoc- sobre el tema de la solidaridad intergeneracional en la familia;

- se trata de una encuesta específica sobre el tema de la solidaridad intergeneracional en la familia, y por tanto que ha explorado muchos aspectos específicos del tema con las herramientas técnicas más adecuadas (baterías de preguntas ya utilizadas en otras investigaciones, nuevas baterías de preguntas, escalas validadas, etc.).

\subsection{Principales resultados}

3.2.1 Estructura intergeneracional de los hogares españoles. Los hogares donde están viviendo dos generaciones juntas son el 25,0\% del total, con tres generaciones el $4,8 \%$, mientras que los restantes casos son hogares de una sola generación (70,2 \%). Las familias donde conviven más generaciones por lo tanto no han desaparecido, y en realidad el 29,8\% representa un dato algo sorprendente.

3.2.2 Relaciones de solidaridad entre padres mayores e hijos adultos.

a) Sentimientos hacia los hijos. Se ha preguntado a los entrevistados sobre los estados de ánimo o los sentimientos que podrían experimentar con respecto a sus hijos. Los 
sentimientos negativos o que indiquen la existencia de una relación negativa o ambivalente (rencor, remordimiento, culpabilidad) han tenido los valores medios más bajos, mientras que los sentimientos positivos (orgullo por los hijos, reciprocidad, gratitud, deseo de compensarlos) han tenido los valores medios más altos (Tabla 1).

La añoranza se experimenta alguna vez. Las mujeres con respecto a los varones experimentan los sentimientos positivos ligeramente más a menudo, mientras que los varones se sienten ligeramente más en defecto que las mujeres ("me siento culpable", "siento remordimientos").

b) Relación con los hijos: flujos de la ayuda dada y ayuda recibida. En relación al afecto, la mayoría de los entrevistados $(69,1 \%)$ considera haber recibido una cantidad igual a la que han dado a sus propios hijos; en relación a la ayuda económica la porcentual baja a un $28,1 \%$; y en cuanto a la asistencia y a los cuidados por enfermedad vuelve a subir a un $56,1 \%$ y repunta hasta al $76,8 \%$ cuando se considera el respeto. La generación target ayuda más económicamente a la siguiente según un $60,2 \%$ de los entrevistados y recibe más respecto de lo que da, según el $16,1 \%$ de los entrevistados.

c) Consenso entre ambas generaciones. El $97,2 \%(34,2 \%$ de acuerdo y $63,0 \%$ totalmente de acuerdo) de los padres considera haber hecho todo lo que era posible para el bienestar de sus hijos. Con la afirmación "creo que mis hijos están contentos de lo que han recibido de mí como padre/ madre" el 93,7\% está de acuerdo $(46,5 \%$ de acuerdo y $47,2 \%$ totalmente de acuerdo). El $97,6 \%$ de los padres opina que los hijos tienen una buena opinión de ellos y el 95,8 \% cree haber sido un buen padre/madre para sus hijos. En general, las mujeres siempre expresan sentimientos más positivos que los varones.

d) Apoyo al desarrollo de los hijos. Se pidió a los entrevistados sobre del apoyo que consideran haber dado a sus hijos para conseguir tres fines: profesión, familia e hijos. El 65,1 \% de los entrevistados considera haber dado mucho apoyo a los hijos para labrarse una profesión, el 30,3\% bastante, el 3,1 \% poco y el 1,5 \% nada. El 35,7 \% considera haber dado mucho apoyo para formar una familia, el $34,7 \%$ bastante, el $17,9 \%$ poco y $11,7 \%$ nada. El 31,4 \% considera haber apoyado bastante a los hijos para tener a su vez hijos, el $28,6 \%$ mucho, el $21,7 \%$ poco y nada el $18,3 \%$.

El apoyo parece concentrarse sobre todo en la toma de decisiones en el campo profesional, y no en el personal en relación a la formación de una familia.

Si observamos los datos distinguiendo las respuestas por sexo, las madres han apoyado a sus hijos más que los padres en todos los aspectos $\mathrm{y}$, con más diferencia para formar una familia $\mathrm{y}$ tener hijos.

Destaca la marcada positividad femenina/ materna hacia los hijos, que se impone en todos los indicadores a la positividad masculina/paterna. Incluso el vivir con o sin los hijos no hace ninguna diferencia. La sensación de haber recibido de los suyos en igual mesura con respecto a cuánto les han dado es ligeramente más fuerte en los que viven con los hijos; de la misma manera, los que viven con los hijos emergen ligeramente más los factores que hacen referencia al rol de los padres en relación con los hijos.

3.2.3 Red familiar y de amistades y ayuda dada y recibida de ellos. Un apartado del cuestionario se ha dedicado a investigar la red familiar y de amistades de los entrevistados y consecuentemente cómo viven la reciprocidad y la solidaridad en sus relaciones.

A la vista de estos datos, es posible apuntar cómo el número de familiares, amigos y vecinos con los cuales se puede contar en caso de necesidad es siempre inferior al número de los mismos con los cuales se mantiene un vínculo importante y auténtico.

Tabla 1

Tipología familiar conviviente (frecuencias absolutas y porcentajes)

\begin{tabular}{|l|c|c|}
\hline \multicolumn{1}{|c|}{ Tipología familiar } & Frecuencia & Porcentaje \\
\hline Pareja con hijos & 85 & 14,0 \\
\hline Pareja sin hijos & 310 & 51,0 \\
\hline Pareja con hijos y nietos & 15 & 2,5 \\
\hline Persona sola & 115 & 18,9 \\
\hline Monoparentales & 57 & 9,4 \\
\hline Otro & 26 & 4,3 \\
\hline Total & 608 & 100,0 \\
\hline
\end{tabular}




\section{СОЦИОЛОГИЯ И СОЦИАЛЬНЫЕ ТЕХНОЛОГИИ}

En cuanto a la ayuda dada y la ayuda recibida, es llamativo que en todo tipo de relación los entrevistados perciben que siempre dan más ayuda de la que reciben (Figura 1).

Es especialmente significativo observar cómo más de un tercio de los entrevistados $(34,2 \%)$ han declarado no haber recibido ningún tipo de ayuda en los doces meses anteriores a la entrevista, y cómo la mitad ha declarado no haber ayudado a nadie en el último año (17,6\%).

Es interesante considerar las jerarquías de la ayuda dada y de la ayuda recibida. Con respecto a la primera, el orden resulta: hijo, hija, nieto, esposa/ pareja, esposo/pareja. Con respecto a la segunda: hija, hijo, esposa/pareja, esposo/pareja, hermana. Por lo tanto, son los hijos in primis, seguidos por el esposo/pareja, a ser los destinatarios de la ayuda y los principales proveedores de la misma, aunque es posible relevar una ligera diferencia de género. La ayuda dada se dirige mayoritariamente hacia los hijos varones más que hacia las hijas, mientras que la ayuda recibida actúa en sentido opuesto: está prestado más por las hijas que por los hijos varones.
Por otra parte, los flujos de ayuda se dirigen de padres a hijos y viceversa, y hacia los nietos, más que en dirección al cónyuge/pareja. La diferencia entre ayuda dada y recibida es mayor en estas relaciones verticales, mientras que tiende a igualarse en las horizontales. Se podría hacer la hipótesis de que en las relaciones donde la ayuda tiene más carga normativa la tendencia es a dar más.

Es interesante comparar la percepción de la ayuda dada y recibida entre esposo/pareja y esposa/pareja. Las mujeres perciben haber dado y recibido ayuda de sus parejas mucho más que los varones. Es decir, las mujeres perciben más la dimensión de mutua ayuda del esposo/pareja.

3.2.3 Actitud hacia las generaciones. A la pregunta sobre la responsabilidad de los padres hacia los hijos (Tabla 2), el 63,8\% de los entrevistados ha contestado que es deber de los padres hacer lo mejor para sus hijos, aunque sea a costa de grandes sacrificios; mientras que el 21,7 \% ha contestado que los padres tienen su vida y no se debería pedirles sacrificios excesivos.

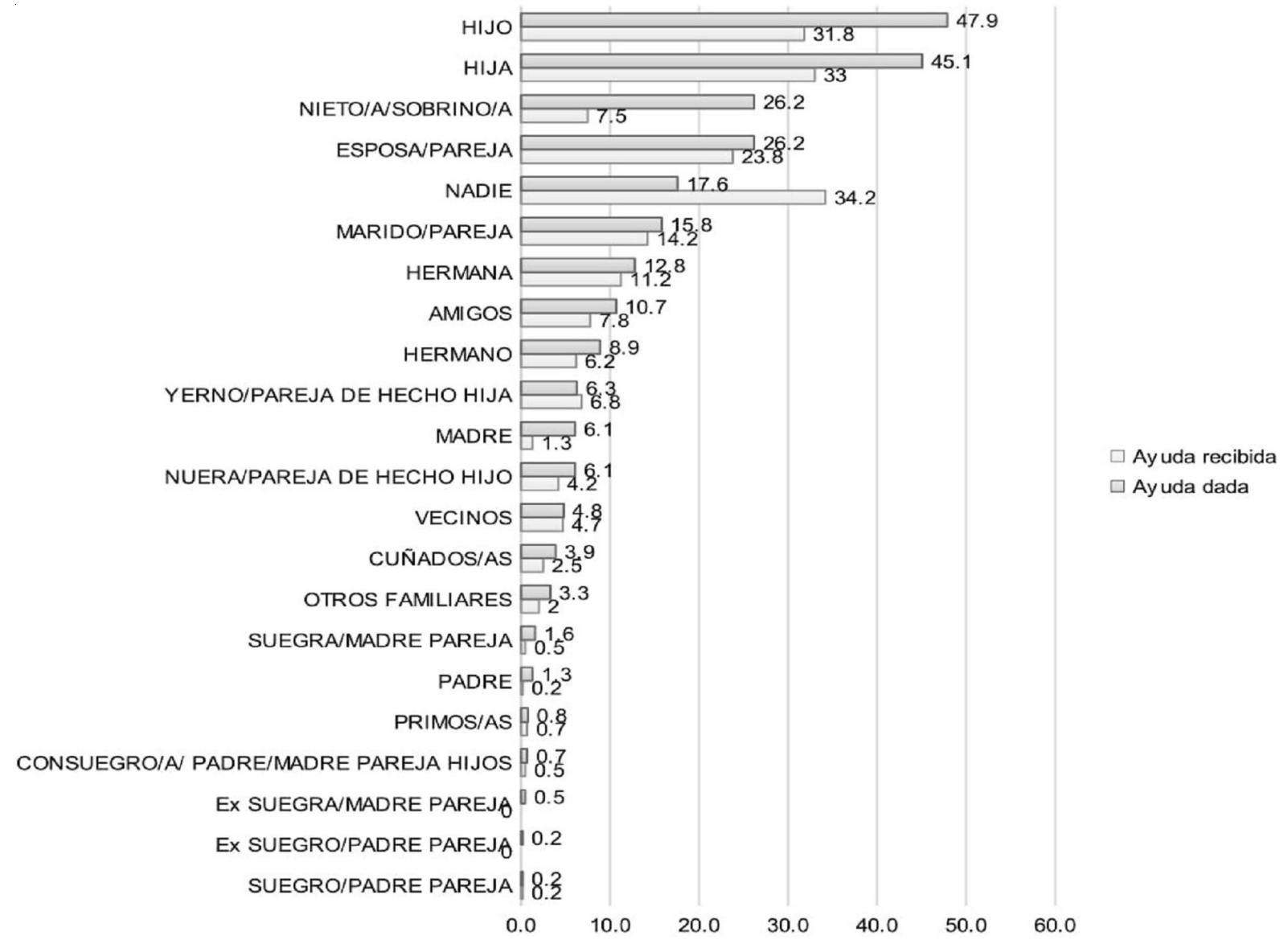

Figura 1. Ayuda dada y ayuda recibida (porcentaje de casos) 
Actitud hacia las generaciones (porcentajes)

\begin{tabular}{|l|c|c|c|c|c|c|c|}
\hline & \multicolumn{2}{|c|}{ Sexo } & \multicolumn{2}{c|}{ Grupos de edad } & \multicolumn{2}{c|}{ Vive con hijos } & Total \\
\cline { 2 - 8 } & Masculino & Femenino & $65-69$ & $70-74$ & No & Sí & \\
\hline $\begin{array}{l}\text { El deber de los padres es } \\
\text { hacer lo mejor para sus } \\
\text { hijos, aunque sea costa } \\
\text { de grandes sacrificios }\end{array}$ & 62,3 & 65,1 & 63,8 & 63,8 & 63,0 & 66,2 & 63,8 \\
\hline $\begin{array}{l}\text { Los padres tienen su vida } \\
\text { y no se les debería pedir } \\
\text { sacrificios excesivos }\end{array}$ & 23,2 & 20,4 & 23,6 & 19,5 & 23,1 & 17,8 & 21,7 \\
\hline $\begin{array}{l}\text { Ninguna de las dos } \\
\text { afirmaciones }\end{array}$ & 12,3 & 13,3 & 11,7 & 14,2 & 12,0 & 15,3 & 12,8 \\
\hline No sé & 2,1 & 1,2 & 0,9 & 2,5 & 2,0 & 0,6 & 1,6 \\
\hline Total & 100,0 & 100,0 & 100,0 & 100,0 & 100,0 & 100,0 & 100,0 \\
\hline
\end{tabular}

A la pregunta sobre la responsabilidad de los hijos hacia los padres (Tabla 3), el 44,9 \% ha contestado que los hijos adultos tienen su vida y no se les debería pedir sacrificios excesivos, mientras que el $33,4 \%$ ha contestado que los hijos adultos tienen el deber de ofrecer asistencia a los padres mayores, aunque sea a costa de grandes sacrificios. Por lo tanto, la solidaridad normativa se percibe más fuerte hacia unos mismos en cuantos padres, de cuanto sea percibida como vinculante para los hijos.

3.2.4 Memoria y gratitud. Una sección del cuestionario ha sido dedicada a los temas de la memoria y de la gratitud, en ambos casos en clave intergeneracional. El punto de partida sigue siendo la percepción de los padres mayores entrevistados, y el tema de la memoria representa el terreno de la trasmisión de la historia familiar a las generaciones sucesivas. El sentido de gratitud concierne la mirada retrospectiva sobre el recorrido de vida realizado, por tanto dirigido al pasado, pero declinado además en relación con los hijos que se han tenido y a los eventuales nietos.

En primer lugar, se ha preguntado a los entrevistados si, en su opinión, los hijos conocían su historia familiar (Tabla 4).

Tabla 3

Actitud hacia las generaciones (porcentajes)

\begin{tabular}{|l|c|c|c|c|c|c|c|}
\hline & \multicolumn{2}{|c|}{ Sexo } & \multicolumn{2}{c|}{ Grupos de edad } & \multicolumn{2}{c|}{ Vive con hijos } & Total \\
\cline { 2 - 8 } & Masculino & Femenino & $65-69$ & $70-74$ & No & Sí & \\
\hline $\begin{array}{l}\text { Los hijos adultos tienen el } \\
\text { deber de ofrecer asistencia } \\
\text { a los padres mayores, }\end{array}$ & 32,7 & 34,0 & 34,0 & 32,6 & 30,8 & 40,8 & 33,4 \\
$\begin{array}{l}\text { aunque sea a costa de } \\
\text { grandes sacrificios }\end{array}$ & & & & & & & \\
\hline $\begin{array}{l}\text { Los hijos adultos tienen su } \\
\text { vida y no se les debería } \\
\text { pedir sacrificios excesivos }\end{array}$ & 44,4 & 45,4 & 45,7 & 44,0 & 47,9 & 36,3 & 44,9 \\
\hline $\begin{array}{l}\text { Ninguna de las dos } \\
\text { afirmaciones }\end{array}$ & 20,4 & 17,3 & 17,2 & 20,6 & 18,0 & 21,0 & 18,8 \\
\hline No sé & 2,5 & 3,4 & 3,1 & 2,8 & 3,3 & 1,9 & 3,0 \\
\hline Total & 100,0 & 100,0 & 100,0 & 100,0 & 100,0 & 100,0 & 100,0 \\
\hline
\end{tabular}

Memoria: ¿Cree que sus hijos conocen su historia familiar? En particular... (Porcentaje de: Mi hijo/a - Todos mis hijos/as)

\begin{tabular}{|l|c|c|}
\hline & Frecuencia & Porcentaje \\
\hline Son capaces de remontarse a sus antepasados: bisabuelos, tatarabuelos & 333 & 54,8 \\
\hline Conocen la historia de sus abuelos & 507 & 83,4 \\
\hline Conocen la historia de ustedes (padre y madre) antes del matrimonio & 545 & 89,6 \\
\hline
\end{tabular}


El conocimiento de los hijos sobre sus antepasados, abuelos y padres, baja a medida que se incrementa la distancia generacional.

Se ha preguntado a los entrevistados expresar su acuerdo/desacuerdo con una serie de afirmaciones relacionadas con sus sentimientos de gratitud hacia personas -especialmente hijos y nietos-, acontecimientos, situaciones... (Tabla 5). Los valores del acuerdo más altos han sido atribuidos a la gratitud hacia los "hijos" (4,65 sobre $5,00)$ y a los "nietos" $(4,61)$, seguidos de "muchas cosas" $(4,29)$, “acontecimientos" $(4,23)$ y "situaciones vitales" $(4,08)$. Los valores medios de las mujeres son siempre mayores que los de los varones.

En el conjunto, los participantes de la encuesta se han mostrado muy agradecidos por lo que la vida le ha dado, especialmente por haber tenidos hijos y, por parte de quien los ha tenido, también por los nietos. Los descendientes, por su parte, son muy partícipes de la historia de la familia, conociendo muy bien, en la mayoría de los casos, los asuntos de los ascendientes.

3.2.5 Capital Social. El capital social representa un tema importante para analizar que la solidaridad intergeneracional tenga conexión con la posesión de recursos relacionales en la convivencia familiar (capital social primario) y en el entorno relacional más amplio (capital social secundario).

La medición del capital social primario se ha hecho a través de preguntas estructuradas sobre la confianza, la ayuda recíproca, la colaboración y el desarrollo de actividades en común (refiriéndose a la familia como a los entrevistados y a sus hijos, aunque no convivan).

Los entrevistados afirman que confían en sus familias $(4,36$ sobre 5$)$ y sienten que pueden contar el uno con el otro $(4,39)$, además de poder expresar libremente ideas y opiniones $(4,27)$. También, se dejan e intercambian objetos personales $(3,64)$.

En cuanto a la ayuda recíproca (Tabla 6), los entrevistados afirman que en su propia familia cada miembro puede contar con los demás para recibir apoyo moral $(4,25$ sobre 5$) \mathrm{y}$, en orden decreciente, si alguien tiene problemas pide ayuda a los demás $(4,10)$, quien ofrece ayuda para algo concreto sabe que los demás también harán lo mismo $(4,08)$, quien da consejos también los acepta $(4,00)$ y, por último, con una puntuación más baja, los entrevistados afirman que en sus familias se exige demasiado a sus miembros $(2,21)$.

Tabla 5

Gratitud (Escala 1-5, donde 1 = totalmente en desacuerdo y 5 = totalmente de acuerdo, valores medios)

\begin{tabular}{|l|c|c|c|}
\hline & Masculino & Femenino & Total \\
\hline Tengo muchas cosas por las que estar agradecido/a en mi vida & 4,23 & 4,35 & 4,29 \\
\hline Siento agradecimiento hacia una amplia variedad de personas & 4,02 & 4,13 & 4,08 \\
\hline Siento mucho agradecimiento a la vida por los hijos que he tenido & 4,63 & 4,66 & 4,65 \\
\hline Siento mucho agradecimiento a la vida por los nietos que he tenido (N. $=496)$ & 4,51 & 4,69 & 4,61 \\
\hline Cuando miro el mundo no veo mucho que agradecer & 2,74 & 2,54 & 2,63 \\
\hline $\begin{array}{l}\text { Si tuviese que hacer una lista de todas las cosas por las que estoy agradecido/a, } \\
\text { sería una lista muy larga }\end{array}$ & 3,81 & 3,98 & 3,90 \\
\hline $\begin{array}{l}\text { Cuanto más pasan los años, me siento más capaz de apreciar las personas, los } \\
\text { acontecimientos y las situaciones que forman parte de la historia de mi vida }\end{array}$ & 4,23 & 4,23 & 4,23 \\
\hline Pasará mucho tiempo hasta que yo sienta agradecimiento hacia algo o alguien & 2,33 & 2,25 & 2,29 \\
\hline
\end{tabular}

Tabla 6

Ayudarse, prestarse ayuda (Escala 1-5, donde $1=$ nada cierto y $5=$ totalmente cierto, valores medios)

\begin{tabular}{|c|c|c|c|}
\hline & Media & \multicolumn{2}{|c|}{ Vivir con los hijos } \\
\hline En cuanto a ayudarse, prestarse apoyo... & & No & Sí \\
\hline $\begin{array}{l}\text { En nuestra familia, quien ofrece una ayuda para algo concreto sabe que los demás } \\
\text { también harán lo mismo }\end{array}$ & 4,08 & 4,06 & 4,14 \\
\hline En nuestra familia, si alguien tiene problemas pide ayuda a los demás & 4,10 & 4,08 & 4,17 \\
\hline En nuestra familia se exige demasiado de los demás & 2,21 & 2,25 & 2,07 \\
\hline En nuestra familia cada miembro puede contar con los demás para recibir apoyo moral & 4,25 & 4,22 & 4,33 \\
\hline En nuestra familia, quien da consejos también los acepta & 4,00 & 3,99 & 4,04 \\
\hline
\end{tabular}


En cuanto a la colaboración y hacer las cosas conjuntamente (Tabla 7) los entrevistados afirman que la educación de los hijos es una cosa compartida por los dos padres $(4,17$ sobre 5$) \mathrm{y}$, en orden decreciente, cuando hay un problema todo el mundo colabora para solucionarlo $(4,01)$, las decisiones se toman entre todos $(3,98)$, todo el mundo echa una manos en las actividades cotidianas $(3,73)$ todo el mundo colabora (según sus capacidades) en las tareas domésticas $(3,69)$, cuando hay un problema se invita a todo el mundo a hacer sugerencias $(3,62)$.

Como es posible observar en la Figura 2, la relación entre capital social primario y solidaridad intergeneracional funcional es positiva: cuanto más elevada es la solidaridad intergeneracional, más alto es el capital social, y viceversa.

3.2.5 La percepción personal y social de la condición de anciano. Entre los entrevistados, solo una minoriìa considera ser una persona mayor, pero se sienten considerados mayores por la sociedad juzga su condicioìn.

Personal: Según las conclusiones de nuestro informe lo que más influye en que una persona se perciba como anciana son las condiciones físicas: el percibir que se pierden las facultades cognitivas; la soledad, y el haber perdido a uno o varios seres queridos.

Social: el trato recibido por otras personas de su familia y amigos, así como la consideración social de ese tramo de la vida, generalmente minusvalorado o invisibilizado. Es importante diseñar modo de hacer partícipes a los mayores de la vida cívica, no sólo por evitar esa percepción sino porque realmente su bagaje vital puede ser una ayuda inestimable. Además, ellos son la memoria, que no debemos ni podemos olvidar como sociedad.

Tabla 7

Colaborar, hacer las cosas conjuntamente

(Escala 1-5, donde $1=$ nada cierto y $5=$ totalmente cierto, valores medios)

\begin{tabular}{|c|c|c|c|}
\hline & Media & \multicolumn{2}{|c|}{ Vivir con los hijos } \\
\hline En cuanto a colaborar, hacer las cosas conjuntamente... & & No & Sí \\
\hline $\begin{array}{l}\text { En nuestra familia la educación de los hijos es una cuestión compartida por ambos } \\
\text { padres }\end{array}$ & 4,17 & 4,14 & 4,25 \\
\hline En nuestra familia las decisiones se toman entre todos & 3,98 & 3,96 & 4,05 \\
\hline En nuestra familia, cuando hay un problema, todo el mundo colabora para resolverlo & 4,01 & 3,99 & 4,08 \\
\hline $\begin{array}{l}\text { En nuestra familia, cuando hay un problema, se invita a todo el mundo a hacer } \\
\text { sugerencias }\end{array}$ & 3,62 & 3,58 & 3,71 \\
\hline $\begin{array}{l}\text { En nuestra familia todo el mundo colabora (según sus capacidades) en las tareas } \\
\text { domésticas }\end{array}$ & 3,69 & 3,69 & 3,70 \\
\hline En nuestra familia todo el mundo echa una mano en las actividades cotidianas & 3,73 & 3,72 & 3,76 \\
\hline
\end{tabular}

4.25

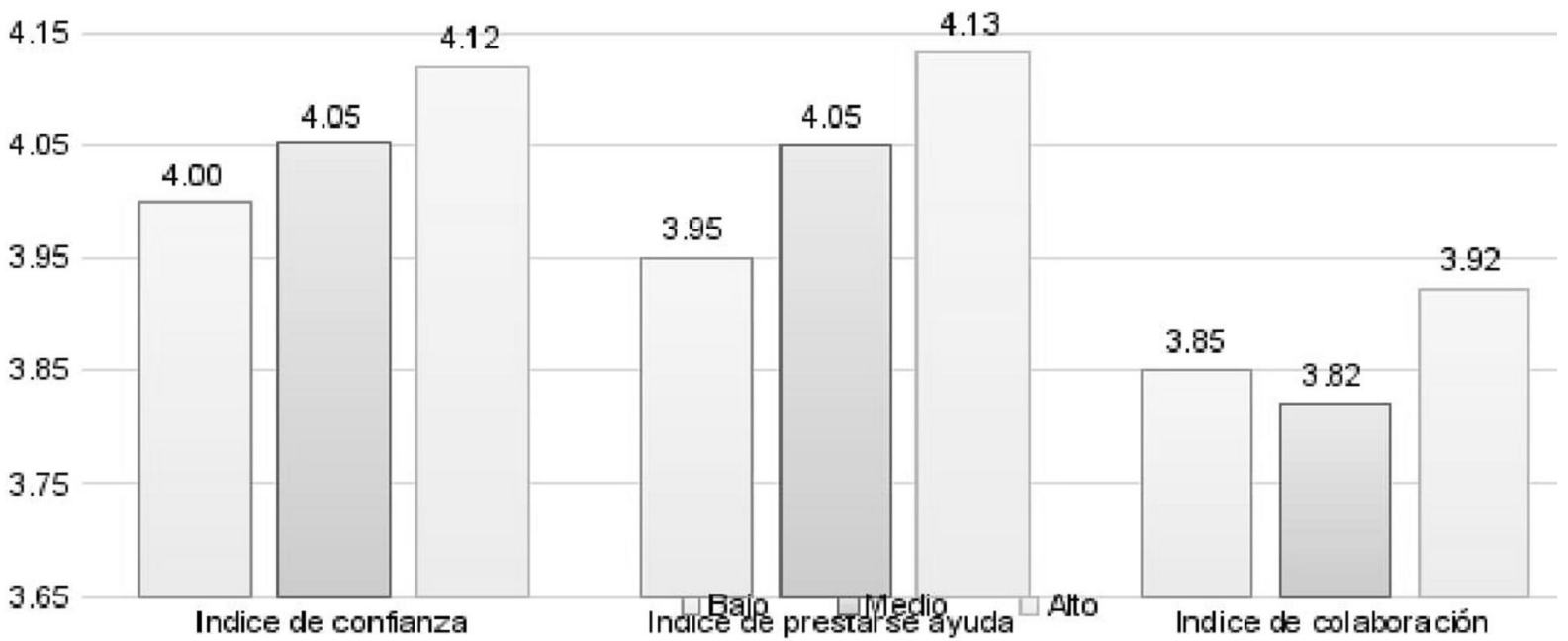

Figura 2. Dimensiones de capital social primario (índice de confianza, índice de prestarse ayuda y índice de colaboración) para niveles (bajo, madio, alto) de ayuda dada a los hijos (valores medios) 


\section{СОЦИОЛОГИЯ И СОЦИАЛЬНЫЕ ТЕХНОЛОГИИ}

Preguntados sobre los aspectos que contribuyen a que una persona se sienta anciana (Figura 3), los entrevistados han indicado en orden decreciente: las condiciones físicas ( $72,9 \%$ de los casos), percibir que se pierden las facultades cognitivas $(44,6 \%)$, la soledad (42,6\%), el haber perdido a uno o varios seres queridos $(19,4 \%)$, la falta de proyectos para el futuro (13,7 \%), el no saber cómo pasar el tiempo $(13,3 \%)$, el haber dejado de trabajar $(9,0 \%)$, las dificultades económicas $(7,9 \%)$, el haber reducido las relaciones sociales $(7,2 \%)$ y el sentirse excluido de las nuevas tecnologías de la comunicación $(2,0 \%)$.

El nivel de satisfacción de los entrevistados (Figura 4), refleja, en orden decreciente los aspectos más determinantes: la familia (valor medio 3,69 sobre una escala de 1 a 4), el lugar donde viven $(3,45)$, su vida en conjunto $(3,43)$, sus amigos $(3,39)$, sus relaciones con los vecinos $(3,30)$, el trabajo que se ha realizado $(3,20)$, el estado de salud $(3,17)$, las metas alcanzadas $(3,17)$, su vida espiritual $(3,05)$ y por último los ingresos familiares $(2,85)$.

\section{$\begin{array}{lllllllll}0.0 & 10.0 & 20.0 & 30.0 & 40.0 & 50.0 & 60.0 & 70.0 & 80.0\end{array}$}

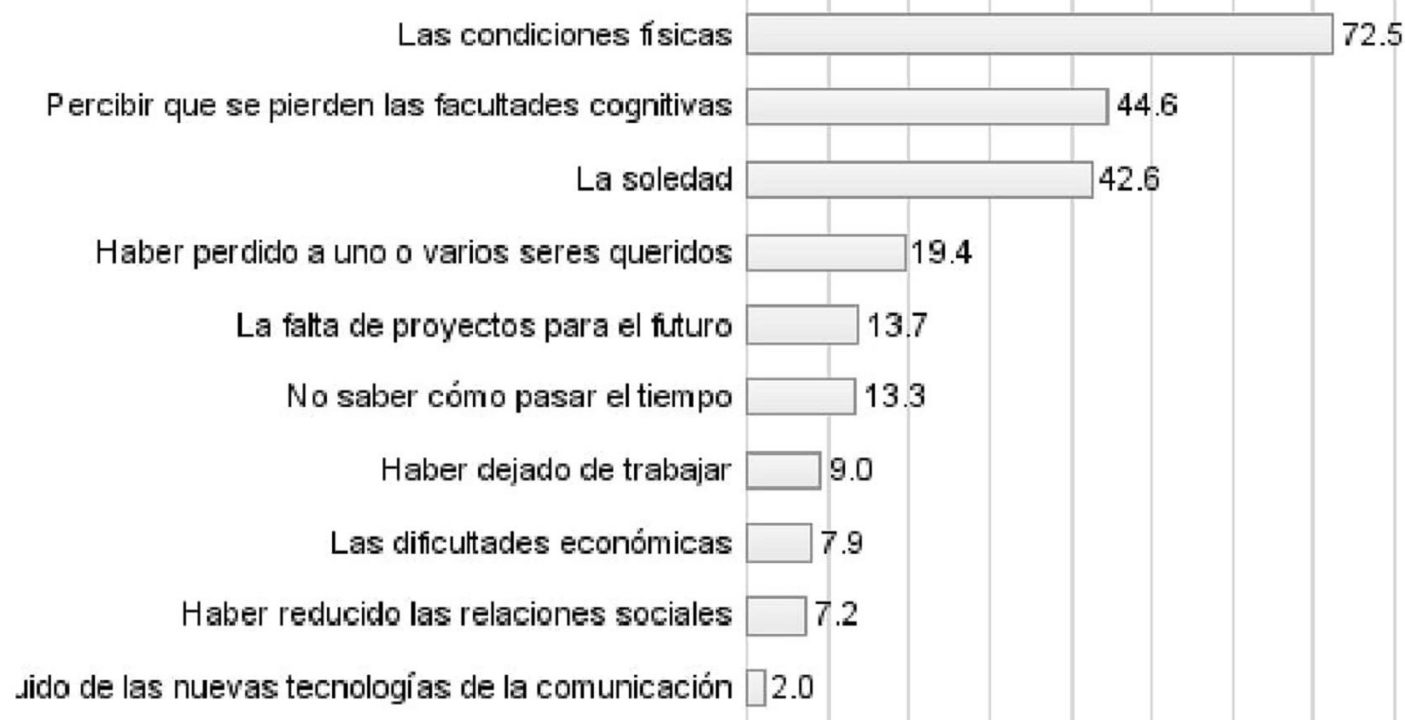

Figura 3. Aspectos que más contribuyen a que una persona se sienta anciana (porcentajes de casos)

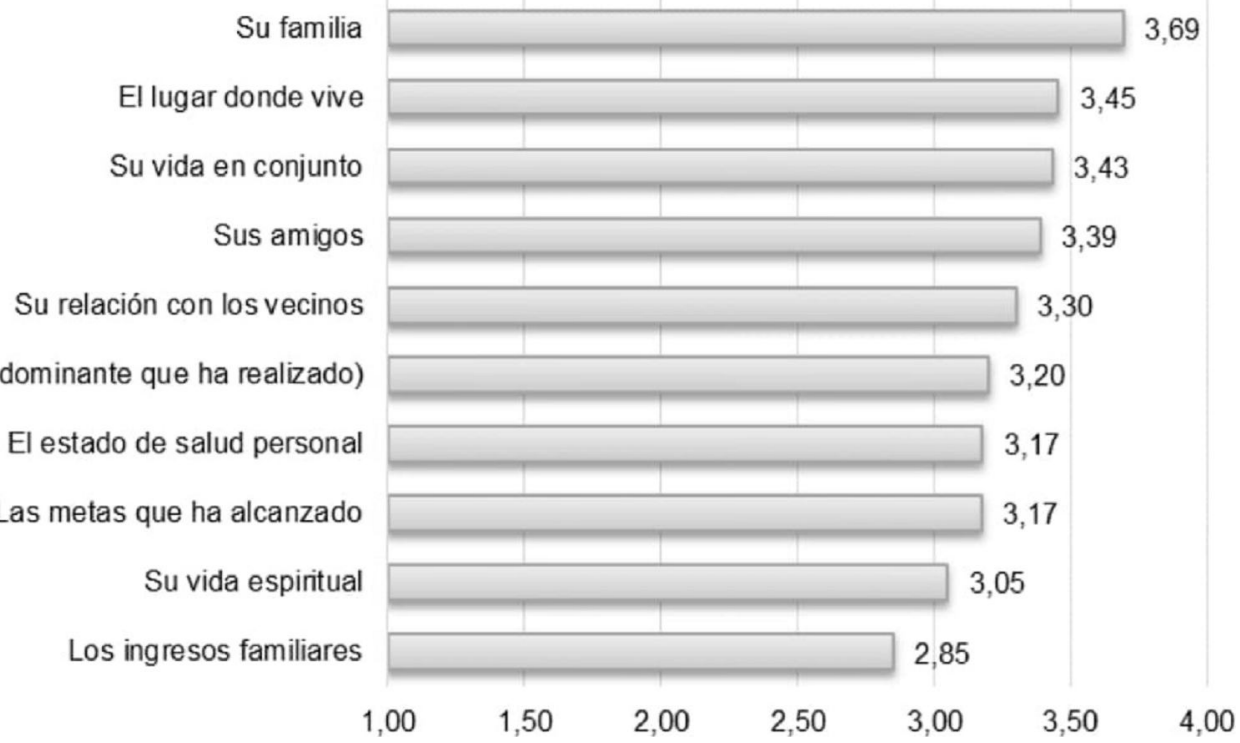

Figura 4. Satisfacción con los siguientes aspectos (Escala $1-4$, donde 1 = nada y $4=$ mucho, valores medios) 
Entre las preocupaciones (Figura 5), las más elevadas hacen referencia al estado de salud y enseguida a las relaciones familiares $y$, especialmente, al ser una carga en la familia y a no tener quién le cuide en un futuro o quedarse solo. Todas las demás preocupaciones resultan más marcadas en el grupo de los que se sienten más ancianos.
3.2.5 Valores de las personas mayores. A los entrevistados le se ha pedido atribuir una puntuación (entre $1=$ nada y $4=$ mucho) a una serie de valores (Figura 6), donde se presentan diferenciados por sexo). Desde el más apreciado al meno apreciado figuran: la familia $(3,85)$, el respeto a los demás $(3,83)$, la responsabilidad $(3,76)$, fidelidad $(3,72)$, la amistad $(3,67)$, la libertad

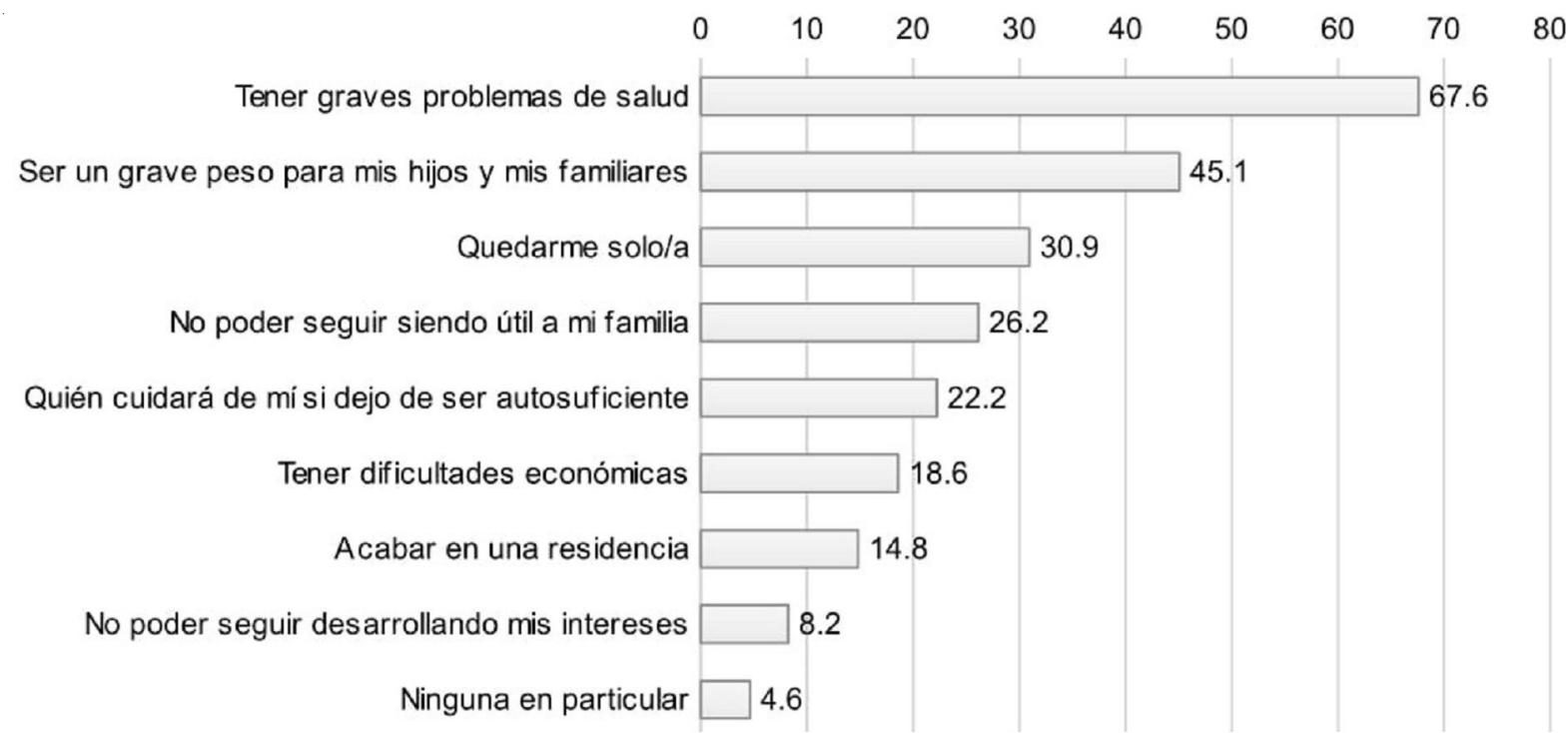

Figura 5. ¿Cuáles son las principales preocupaciones en relación al futuro? (porcentajes de casos)

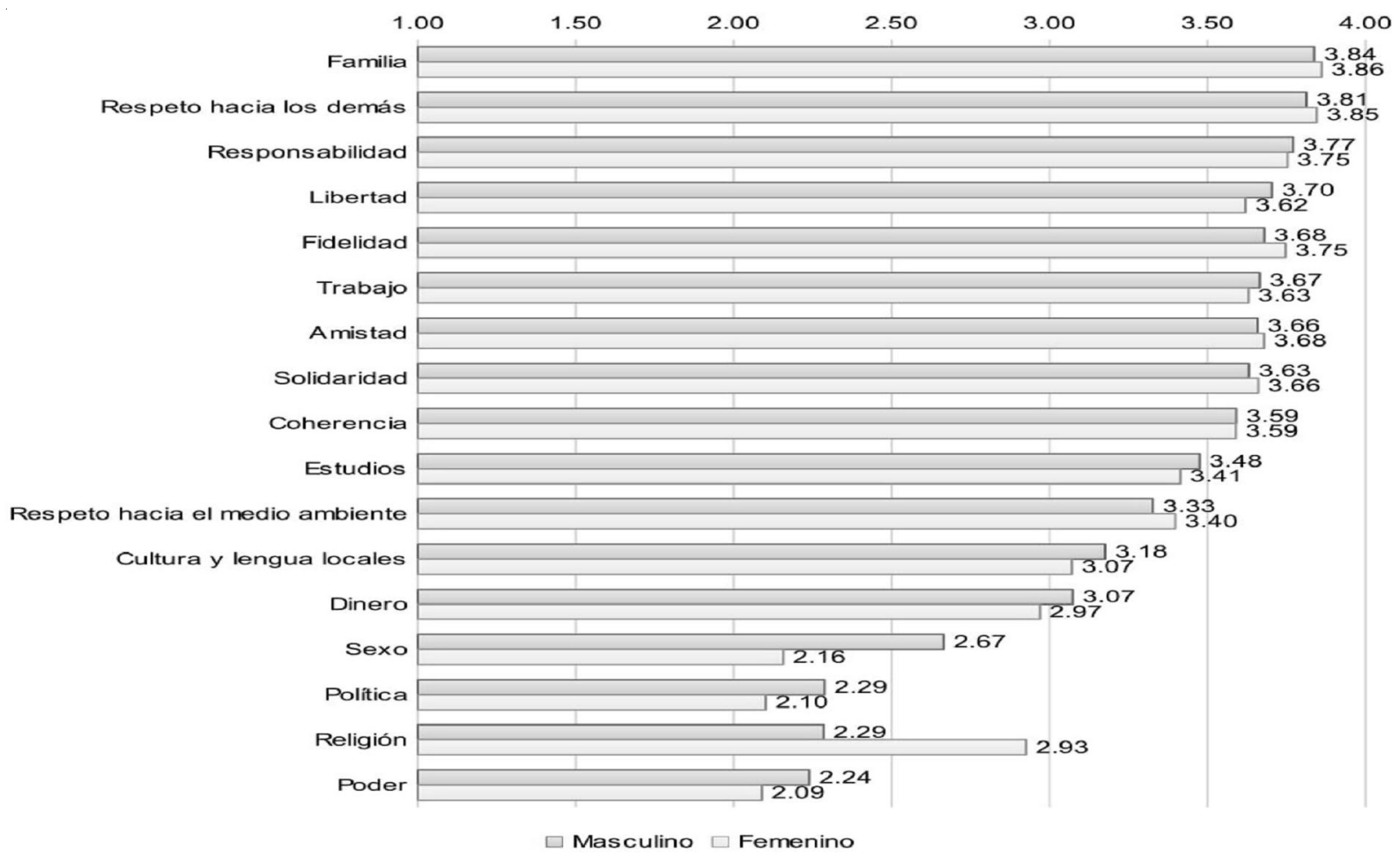

Figura 6. En qué medida son importantes los siguientes valores

(Escala 1-4, donde 1 = nada y 4 = mucho, valores medios) 


\section{СОЦИОЛОГИЯ И СОЦИАЛЬНЫЕ ТЕХНОЛОГИИ}

$(3,66)$, el trabajo $(3,65)$, la solidaridad $(3,65)$, la coherencia $(3,59)$, los estudios $(3,44)$, el respeto hacia al medio ambiente $(3,37)$, la cultura y la lengua locales $(3,12)$, el dinero $(3,02)$, la religión $(2,63)$, el sexo $(2,39)$, la política $(2,19)$ y el poder $(2,16)$. Se aprecian diferencias entre los sexos en relación a los cuatros últimos valores. Las mujeres valoran mucho más la religión $(2,93)$, mientras que los hombres, aunque de manera menos acentuada, valoran más el sexo $(2,67)$, la política $(2,29)$ y el poder $(2,24)$.

\section{Discusión de los resultados}

Las 608 respuestas de la muestra representativa de la población de España de esta clase de edad, constata que la vinculación real en el tiempo y en el espacio de las diferentes generaciones presentes simultáneamente refuerza estas dimensiones y hace más fácil la solidaridad intergeneracional.

En España predomina de manera clara un sentimiento positivo de los padres hacia los hijos en términos de orgullo y gratitud (Tabla 1). También es mayoritario el sentimiento de gratitud hacia los hijos y nietos, por encima de otras personas o valores de la vida (Tabla 5).
También hay un muy alto grado de satisfacción de los padres hacia el cumplimiento de sus deberes como padres y madres y al haber satisfecho las expectativas de sus hijos respecto a ellos (Tabla 8).

A pesar de ello, el apoyo de los padres entrevistados a sus hijos se ha centrado en los aspectos profesionales, prestando una atención menor al apoyo para formar una familia y tener hijos. Se trata sin duda de un factor que ha influido en la creciente inestabilidad de las familias en España y en el bajo índice de natalidad que experimentamos.

Se produce un alto nivel de correspondencia por parte de los hijos, sobre todo en términos de afecto y de respeto; y en menor medida en términos de ayuda en caso de enfermedad y apoyo económico (Tabla 9).

En general, las personas mayores en España sienten que han prestado más ayuda de la que han recibido de otros (Figura 1). Esto nos lleva a pensar que en términos de cuidado las jóvenes generaciones tienen menor capacidad de atender a sus mayores.

En cuanto a la solidaridad normativa, es mayoritaria en esta generación de mayores la

Tabla 8

Grado de acuerdo con las afirmaciones (Escala 1-7,

donde $1=$ totalmente en desacuerdo y $7=$ totalmente de acuerdo, valores medios)

\begin{tabular}{|l|c|c|c|c|c|c|c|}
\hline & \multicolumn{2}{|c|}{ Sexo } & \multicolumn{2}{c|}{ Grupos de edad } & \multicolumn{2}{c|}{ Vive con hijos } & \multirow{2}{*}{ Total } \\
\cline { 2 - 6 } & Masculino & Femenino & $65-69$ & $70-74$ & No & Sí & \\
\hline $\begin{array}{l}\text { Creo que mis hijos habrían querido que yo fuese un(a) } \\
\text { padre/madre distinto/a }\end{array}$ & 3,08 & 2,86 & 2,90 & 3,04 & 2,92 & 3,08 & 2,96 \\
\hline $\begin{array}{l}\text { Creo haber hecho todo lo que era posible por el } \\
\text { bienestar de mis hijos }\end{array}$ & 6,47 & 6,54 & 6,50 & 6,51 & 6,50 & 6,54 & 6,51 \\
\hline $\begin{array}{l}\text { Creo que mis hijos están contentos de lo que han } \\
\text { recibido de mí como padre/madre }\end{array}$ & 6,12 & 6,33 & 6,24 & 6,22 & 6,19 & 6,35 & 6,23 \\
\hline $\begin{array}{l}\text { En general creo haberme comportado de forma } \\
\text { correcta con mis hijos }\end{array}$ & 6,39 & 6,49 & 6,44 & 6,44 & 6,42 & 6,51 & 6,44 \\
\hline Creo que mis hijos tienen una buena opinión de mí & 6,13 & 6,37 & 6,24 & 6,28 & 6,21 & 6,39 & 6,26 \\
\hline $\begin{array}{l}\text { Si tengo que hacer balance hasta hoy, creo haber sido } \\
\text { en general un(a) buen(a) padre/madre para mis hijos }\end{array}$ & 6,25 & 6,40 & 6,34 & 6,32 & 6,30 & 6,41 & 6,33 \\
\hline
\end{tabular}

Tabla 9

Lo que he recibido de mis hijos es... (Escala 1-7, donde $1=$ he recibido de mis hijos mucho más, $4=$ he dado y recibido en igual medida y $7=$ he dado a mis hijos mucho más, valores medios)

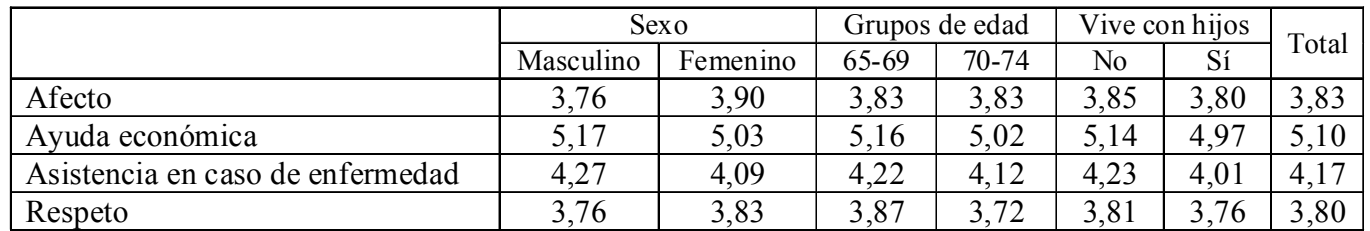


percepción del deber de hacer lo mejor para sus hijos a pesar de los sacrificios (Tabla 2), mientras no consideran en la misma proporción que los hijos tengan este deber respecto a ellos.

Respecto al capital social, nuestro estudio constata también algo que quizás de modo intuitivo ya sabíamos: las personas mayores que viven solas son las que se perciben a sí mismas como más ancianas, tienen peor estado de salud, están menos activas, son más pesimistas y muestran más insatisfacción vital. En estas personas además coincide que su red familiar y de amistad es menos estrecha, los intercambios menos intensos y frecuentes, y el capital social muy bajo. Se puede afirmar que "vivir solos amplifica la condición anciana y está más asociado al riesgo de pérdida de autonomía personal". El motivo por el que esto es así no se reduce a uno. Algunas personas no han tenido hijos, otras han tenido sólo uno y algunas tienen a sus hijos en otro país. Por otra parte, nuestra sociedad ha sufrido un proceso creciente de individualización y en ocasiones se ha maximizado la autonomía como un valor absoluto cuando en realidad somos seres interdependientes y nos necesitamos, mucho más en el último tramo vital.

En relación a la percepción de sentirse mayor, de acuerdo a los resultados de nuestro estudio, lo que más influye en que una persona se perciba como anciana son las condiciones físicas: la pérdida de las facultades cognitivas; pero también la soledad, y el haber perdido a uno o varios seres queridos, la falta de proyectos para el futuro y el no saber cómo pasar el tiempo.

Entre las preocupaciones (Figura 4), las más elevadas hacen referencia al estado de salud y enseguida a las relaciones familiares $y$, especialmente, al ser una carga en la familia y a no tener quién le cuide en un futuro o quedarse solo.

Finalmente cabe hacer referencia al nivel de satisfacción global de los entrevistados (Figura 4) y a sus valores (Figura 5). Se refleja un hecho muy relevante: la familia es la primera fuente de satisfacción, por encima de otros aspectos, sobre todo los materiales y económicos. También es el valor que aparece en primer lugar en la escala. La mayor parte de los valores que aparecen en los primeros lugares son bienes no materiales, mientras los últimos son el dinero, el poder y el sexo, de contenido más material. Esto muestra una prevalencia del primer tipo de valores entre los miembros de esta generación. Cabe comentar sin embargo, que la espiritualidad y la religión no quedan entre los primeros puestos. Esto podría ser un indicador de la secularización de la sociedad española en los últimos 50 años. La religión institucionalizada ha perdido peso. Pero no los valores morales que la religión contiene (solidaridad, respeto, fidelidad, responsabilidad, familia, etc.).

Un análisis de los resultados en función del sexo de los entrevistados, nos llevaría a afirmar que las mujeres son en general más proclives a mantener relaciones de solidaridad intergeneracional, con valores siempre más altos y más positivos. Destaca sobre todo el apoyo afectivo y el apoyo a los hijos para formar su propia familia. Es importante también la mayor percepción por parte de las mujeres de haber recibido correspondencia por parte de los hijos. Del mismo modo, son las hijas las que más ayudan a los padres, mientras la ayuda de los padres y madres es mayor en el caso de los hijos de sexo masculino. El nivel de agradecimiento por los hijos y nietos es también sensiblemente superior al de los varones.

\section{Recomendaciones derivadas}

1. La evolución del envejecimiento de la población requiere el esfuerzo conjunto de todos los componentes de la sociedad (instituciones públicas, sociedad civil, tercer sector, familias) e implica una actitud positiva hacia la innovación social.

2. El papel reconocido a la familia como el entorno natural del cuidado de sus miembros debe ser ampliado y promovido adecuadamente por parte de todos los sectores sociales, especialmente por parte de los organismos públicos (Gobierno).

3. Por lo tanto, las políticas familiares deberían mejorarse e integrarse mejor con las políticas de protección social, de salud pública, del empleo.

4. Se debe potenciar la solidez de la red familiar. Nuestra sociedad ha sufrido un proceso creciente de individualización y en ocasiones se ha maximizado la autonomía como un valor absoluto cuando en realidad somos seres interdependientes y nos necesitamos, mucho más en el último tramo vital.

5. Fomentar las conexiones en la red familiar y de amistad, los intercambios intensos y frecuentes, y diseñar medidas para aumentar el capital social primario y secundario de las personas. 
6. Diseñar modos de hacer partícipes a los mayores de la vida cívica, no sólo por evitar la percepción de que no están plenamente insertados en la sociedad, sino porque realmente su bagaje vital puede ser una ayuda inestimable. Además, ellos son la memoria, que no debemos ni podemos olvidar como sociedad.

\section{ПРИМЕЧАНИЕ}

1 Более полное представление о результатах исследования можно получить, обратившись к публикации Rita Cavallotti, Francesco Marcaletti Older Parents, Generations and Family Solidarity. A Multilevel Analysis of the Spanish Conte. 2018. Instituto de Estudios Superior de la Familia Universitat Internacional de Catalunya Calle Inmaculada, BARCELONA. Available at: https://www.uic.es/sites/ default/files/en_older_parents_generations_and_ family_solidarity.pdf(примечание редколлегии).

\section{REFERENCES}

1. Arber, S., Attias-Donfut, C. The myth of generational conflict: the family and state in ageing societies. - London : Routledge, 2000. 244 p.

2. Bengtson, V. L., Roberts, R.E.L. Intergenerational Solidarity in Aging Families an example of Formal Theory Construction. Journal of Marriage and Family, 1991, no. 53 (4), pp. 856-870.

3. Binstock, R. H. From Compassionate Ageism to Intergenerational Conflict? The Gerontologist, 2010, no. 50 (5), pp. 574-585.
4. Donati, P. Sociologia della relazione. Bologna: Il Mulino, 2013. 183 p.

5. Donati, P. Intergenerational Solidarity: Old and New Scenarios, Challenges and Prospects, in Sustainable Humanity, Sustainable Nature, Our Responsibility. Proceedings of the Pontifical Academy of Sciences/Pontifical Academy of Social Sciences Tenth Plenary Session (Vatican City, 2-6 May 2014). Editors: P. S. Dasgupta, V. Ramanathan and M. Sánchez Sorondo. Vatican City : The Pontifical Academy of Sciences, 2015, pp. 569-613.

6. Jönsson, I. Policy Perspectives on Changing Intergenerational Relations, Journal of Social Policy and Society, 2003, no. 2 (3), pp. 241-248.

7. López López, M.T. Personas mayores y solidaridad intergeneracional en la familia. El caso español, Madrid: Colección Acción Familiar, Ediciones Cinca, 2015. 170 p.

8. Lüscher, K., Sanchez, M., KlimczukA. “Family Structures and Intergenerational Relationships in the Konstanz Region" (Documento de trabajo No. 34.4), Konstanz : Konstanz Universität, 2000.

9. Meil, G. "Individualización y solidaridad familiar”, Colección Estudios Sociales, n.32, Barcelona: Obra Social "La Caixa", 2011. Available at: https://www.tendencias21.net/libros/attachment/ 304211/ (appeal date: 20.08.2018).

10. Scabini E., Rossi G. L'allungamento della vita. Una risorsa per la famiglia, un'opportunità per la società. Milano, Vita e Pensiero, 2016. 325 p.

11. World Health Organisation, Active ageing. A policy framework. Geneva, 2002. Available at: http:// apps.who.int/iris/bitstream/handle/10665/67215/ WHO_NMH_NPH_02.8.pdf;jsessionid=8BA743516DE8 B8B0C D6BEEB2D-D057AE7?sequence $=1$ (appeal date: 08.09.2018).

\section{Information about the Authors}

Belén Zárate Rivero, Doctor of Science (Law), Full Professor of Law, Director of Law's Philosophy Department, International University of Catalonia (UIC Barcelona), Immaculada St., 22, 08017 Barcelona, Spain, bzarate@uic.es.

Montserrat Gas-Aixendri, Doctor of Science (Law), Full Professor of Law, Director of the Institute for Advanced Family Studies, International University of Catalonia (UIC Barcelona), Immaculada St., 22, 08017 Barcelona, Spain, mgas@uic.es.

\section{Информация об авторах}

Белен Зарате Риверо, доктор юридических наук, профессор права, заведующая кафедрой философии права, Международный Университет Каталонии, ул. Иммакулада, 22, 08017 г. Барселона, Испания, bzarate@uic.es.

Монсеррат Гас-Айксендри, доктор юридических наук, профессор права, директор Института углубленного исследования семьи, Международный Университет Каталонии, ул. Иммакулада, 22, 08017 г. Барселона, Испания, mgas@uic.es. 


\section{Acerca de los Autores}

Belén Zárate Rivero, Doctor en Ciencias (Derecho), Profesor titular de derecho, Director del Departamento de Filosofía del Derecho, Universitat Internacional de Catalunya (Barcelona, España), Immaculada Cl., 22, 08017 Barcelona, España, bzarate@uic.es.

Montserrat Gas-Aixendri, Doctor en Ciencias (Derecho), Profesor titular de derecho, Director del Instituto de Estudios Superiores de la Familia, Universitat Internacional de Catalunya (Barcelona, España), Immaculada Cl., 22, 08017 Barcelona, España, mgas@uic.es. 